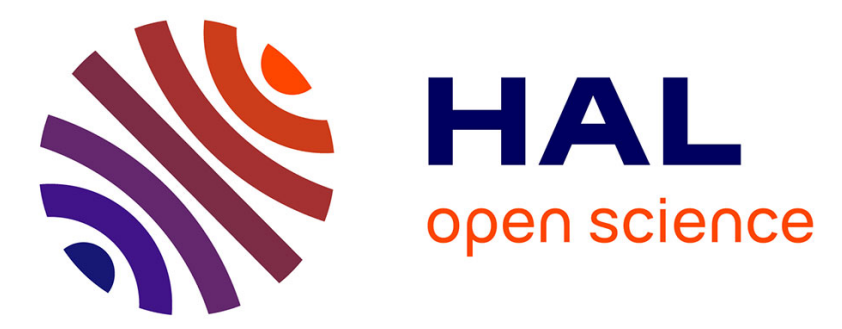

\title{
Attachment cross sections of protonated water clusters
}

Sébastien Zamith, Pierre Feiden, Pierre Labastie, Jean-Marc L'Hermite

\section{To cite this version:}

Sébastien Zamith, Pierre Feiden, Pierre Labastie, Jean-Marc L'Hermite. Attachment cross sections of protonated water clusters. Journal of Chemical Physics, 2010, 133 (15), pp.154305. $10.1063 / 1.3505302$. hal-00527860

\section{HAL Id: hal-00527860 \\ https://hal.science/hal-00527860}

Submitted on 20 Oct 2010

HAL is a multi-disciplinary open access archive for the deposit and dissemination of scientific research documents, whether they are published or not. The documents may come from teaching and research institutions in France or abroad, or from public or private research centers.
L'archive ouverte pluridisciplinaire HAL, est destinée au dépôt et à la diffusion de documents scientifiques de niveau recherche, publiés ou non, émanant des établissements d'enseignement et de recherche français ou étrangers, des laboratoires publics ou privés. 


\section{Attachment cross sections of protonated water clusters}

Sébastien Zamith ${ }^{1,2}$, $)$ Pierre Feiden ${ }^{1,2}$, Pierre Labastie ${ }^{1,2}$, and Jean-Marc L'Hermite ${ }^{1,2}$

${ }^{1}$ Université de Toulouse ; UPS ; Laboratoire collisions Agrégats Réactivité, IRSAMC ; F-31062 Toulouse, France

${ }^{2}$ CNRS ; UMR 5589 ; F-31062 Toulouse, France

(Dated: 4 October 2010)

The attachment of water molecules onto size selected protonated water clusters has been experimentally investigated. Absolute attachment cross sections are measured as a function of cluster size, collision energy and initial cluster temperature. Although thermal evaporation is ruled out in our experiment, attachment cross sections become significantly smaller than hard sphere cross sections as the collision energy increases. This feature is attributed to a transition from adiabatic to non adiabatic regime. It is shown to be due to a dynamical effect: as the collision duration becomes shorter than the typical time required for collision energy redistribution into clusters internal energy, the attachment probability is reduced. We relate this typical time to the period of the main surface vibrational mode excited by the collisions. This hypothesis is further supported by results obtained with deuterated water clusters.

PACS numbers: 36.40.-c, 36.40.Jn, 34.10.+x

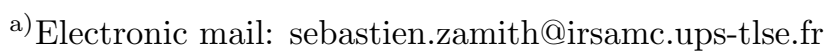




\section{INTRODUCTION}

How droplets are formed from the vapor phase, starting from single molecules or atoms, is still not well known. Nucleation theories, which deal with this issue from the statistical point of view ${ }^{1-4}$, are still unsuccessful in predicting quantitatively the rate of formation of droplets in a vapor ${ }^{5}$. The poor theoretical description and experimental knowledge of particle growth at the molecular scale contributes, among many other barriers to quantitative predictions ${ }^{5,6}$, to the quantitative failure of nucleation theories. In particular, parameters whose knowledge is obviously required and which are not well documented are the attachment cross sections of impinging atoms or molecules onto clusters, and their size scaling laws ${ }^{2,3}$.

The first accurate measurements of absolute attachment cross sections of single atoms onto size selected clusters, over an extended size range in order to determine size scaling laws, has been carried out only recently for sodium clusters ${ }^{7}$. These measurements have been now extended to water, probably the most important chemical compound in nature.

This paper is a follow up of a recent Letter ${ }^{8}$ in which we presented experimental investigations on the attachment properties of water clusters at the molecular level. The attachment cross sections of water molecules onto clusters have been measured as a function of cluster size. The attachment scaling law reveals that water clusters behave approximately as hard spheres from size of about a hundred molecules, whereas at smaller sizes the attachment cross sections are smaller than hard sphere (geometric) cross sections. This was not expected since any electrostatic model predicts that attachment cross sections are greater than geometric ones at low collision energy. This behavior is rationalized by taking into account events where head-on collisions do not lead to attachment. This phenomenon, due to clusters bouncing the impinging molecule off their surface, is shown to be related to the period of the main surface vibration mode of the cluster: when the collision is too short, the vibration of the cluster has not enough time to be excited, thus the collision energy cannot be absorbed by the internal vibrational degrees of freedom of the cluster. In ref. 8 we extracted the time required to excite the clusters surface vibration from our measurements; this time was deduced to be about $0.75 \mathrm{ps}$, which corresponds to a vibration energy of $5.6 \mathrm{meV}$, in very good accordance with the measurement of Brudermann and coworkers who identified the main mode excited in collisions of He with water clusters as the $\mathrm{O} \cdot \cdot \mathrm{O} \cdot \cdot \mathrm{O}$ bending mode at about $5 \mathrm{meV}^{9}$. 


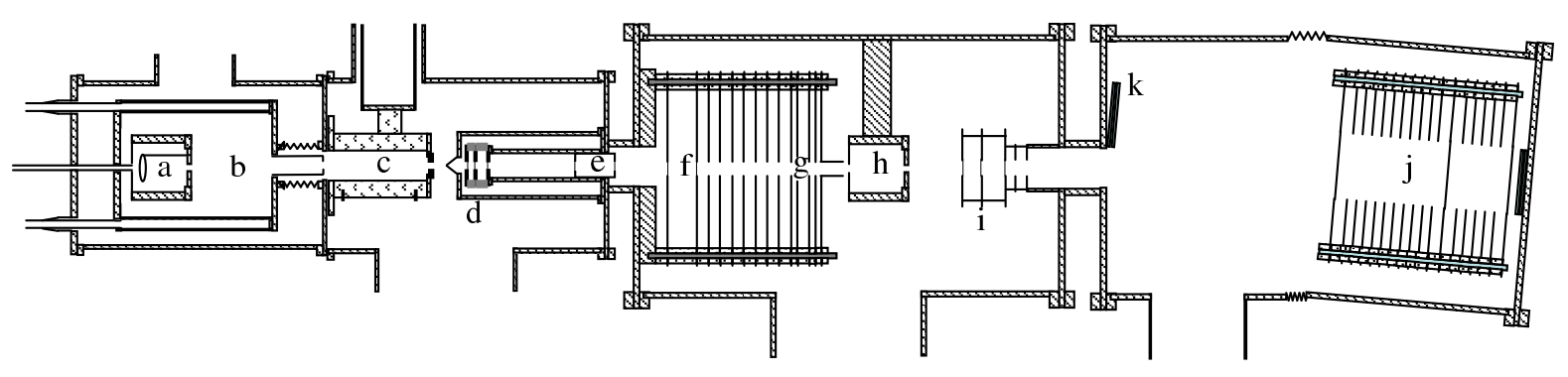

FIG. 1. Overview of the experimental setup. Source chamber (a), Nucleation cell (b), Thermalization (c), First acceleration stage for mass selection (d), Mass filter (e), Energy focussing (f), Deceleration stage (g), Collision cell (h), Acceleration stage for products mass analysis (i), Reflectron (j) and MCP detector (k).

Measuring attachment cross sections and size scaling laws requires being able to attach single molecules or atoms onto mass selected clusters, ideally without subsequent thermal evaporation in order to discriminate both phenomena. Such an experiment has been developed a few years ago in our group ${ }^{10}$. It allows measuring absolute attachment cross sections of atoms or molecules onto mass selected clusters, independently of thermal evaporation. This is ensured by achieving very low collision energy so that the lifetime of the clusters, even after they have undergone attachment, is much larger than their time-of-flight up to the detector.

We will describe in this paper the method used to determine attachment cross section of molecules onto size selected clusters, then we will present and analyze the attachment of water and deuterated water molecules onto protonated water clusters and deuterated water clusters. In section II, the experimental setup is described. Section III is devoted to the presentation of the method employed to measure the cross sections. The experimental results are presented and discussed in section IV. Conclusions will be drawn in section V.

\section{EXPERIMENTAL SETUP}

Apart from the cluster source, the experimental setup is nearly the same as the one used to perform attachment experiments on sodium clusters ${ }^{7,10}$. In the following we will briefly describe the key points of the experimental setup depicted in figure 1. 


\section{A. Cluster production and thermalization}

The positively charged, protonated water clusters are produced in a gas aggregation source. It consists of a double walled chamber (b) in which a smaller cell (a) is housed (in the following the letters between parenthesis refer to figure 1).

A small amount of water vapor is mixed with the helium carrier gas before entering the cell in the source chamber. The amount of water is controlled by a needle valve whereas a flow-meter controls the helium flow. The helium gas seeded with a small amount of water vapor is injected in the cell through a $4 \mathrm{~mm}$ inner diameter stainless steel pipe. The injection pipe is kept above $0{ }^{\circ} \mathrm{C}$ in order to avoid the water vapor freezing. The cell has a diameter of $30 \mathrm{~mm}$ and is $50 \mathrm{~mm}$ long with a $3 \mathrm{~mm}$ diameter output hole. The outer wall of the source (b) is maintained at liquid nitrogen temperature. A heating element controls the cell temperature, generally operated between $233 \mathrm{~K}$ and $273 \mathrm{~K}$ whereas outside the cell the gas temperature is constant at $125 \mathrm{~K}$. The cell position within the source chamber can be longitudinally adjusted within few centimeters.

Within the cell a discharge is established in order to produce charged clusters, both negative and positive. The discharge takes place by applying a negative voltage to a small ring electrode. The voltage applied on the electrode is $-600 \mathrm{~V}$ and results in a current discharge of the order of $100 \mu \mathrm{A}$.

After the cell, clusters grow and propagate in the main chamber. The transfer towards the thermalization chamber takes place through a $18 \mathrm{~mm}$ inner diameter pipe terminated with a $5 \mathrm{~mm}$ diameter hole. The working temperature at the end of this pipe, which is monitored, is $140 \mathrm{~K}$.

After their growth, the clusters enter the thermalization chamber (c). The pressure in the source chamber and thermalization chamber is of the order of 1 mbar. The thermalization chamber consists in a $20 \mathrm{~cm}$ long, $2 \mathrm{~cm}$ inner diameter copper piece. It is attached to a closed-cycle helium cryostat whose temperature $T_{t h}$ is controlled within $1 \mathrm{~K}$ and can be varied from 25 to $340 \mathrm{~K}$. Thermalization occurs trough collisions between the helium carrier gas atoms and the clusters. The estimated number of collisions is of the order of $10^{5}-10^{6}$ which is far enough to completely thermalize the clusters. The output hole of the thermalization chamber is a diaphragm iris whose diameter can be adjusted. The typical value is $5 \mathrm{~mm}$.

We have no detailed explanation on how the cluster growth takes place and the actual 
configuration of the source is a result of a lengthy trial and errors procedure. Nevertheless the exploration of the source parameters gives us general trends. The clusters size-distribution can be tuned by changing the source parameters in a rather reproducible way. For instance the size distribution can be shifted by adjusting the helium flow, the thermalization output hole, the cell temperature or the amount of water. If small sizes are required, a high flow rate and large aperture is desirable: the residence time in the source is shortened and thus their growth. To shorten the growth time one can also move the cell towards the exit of the source chamber. In order to get bigger (smaller) sizes, one can also increase (decrease) the amount of water and/or decrease (increase) the cell temperature . Decreasing (increasing) the cell temperature locally decreases (increases) the water partial pressure (the colder the cell, the more water is deposited on the cell walls) which limits the growth.

We present in figure 2 examples of mass spectra obtained under three different source conditions. In the examples shown in fig. 2(a) we have varied the amount of water to obtain different size distributions. Figure 2(b) shows in more details the structure of the individual peaks in the mass spectrum. The mass resolution is high enough to identify peaks due to the natural isotopes of oxygen $\left({ }^{17} \mathrm{O}\right.$ and ${ }^{18} \mathrm{O}$ mainly).

The count rate on the detector is about $10^{4}$ ions. $\mathrm{s}^{-1}$ for one given size.

\section{B. Mass selection and slowing down}

The mass selection and slowing down of the clusters is detailed in ref. 10 and will only be briefly described here. The only change since our method has been published is the adjunction of a mass filter (e), that is described below, between the first acceleration stage (d) and the energy focussing (f) and slowing down device (g).

The continuous cluster beam enters the first acceleration region (d) through a $1 \mathrm{~mm}$ diameter skimmer. The acceleration of the charged clusters is devoted to a Wiley-Mc Laren type arrangement of three circular electrodes. Two pulsed voltages $V_{1}$ and $V_{2}$ are applied simultaneously to the first two electrodes while the third one is grounded. In the following field free region, the clusters have acquired a kinetic energy dispersion due to their spatial distribution in the acceleration region. The applied voltages are such that when the clusters arrive in a given region (f) they have again nearly linear kinetic energy dispersion. This dispersion can then be compensated by applying at a right time a pulsed voltage $V_{f o c}$ on the 

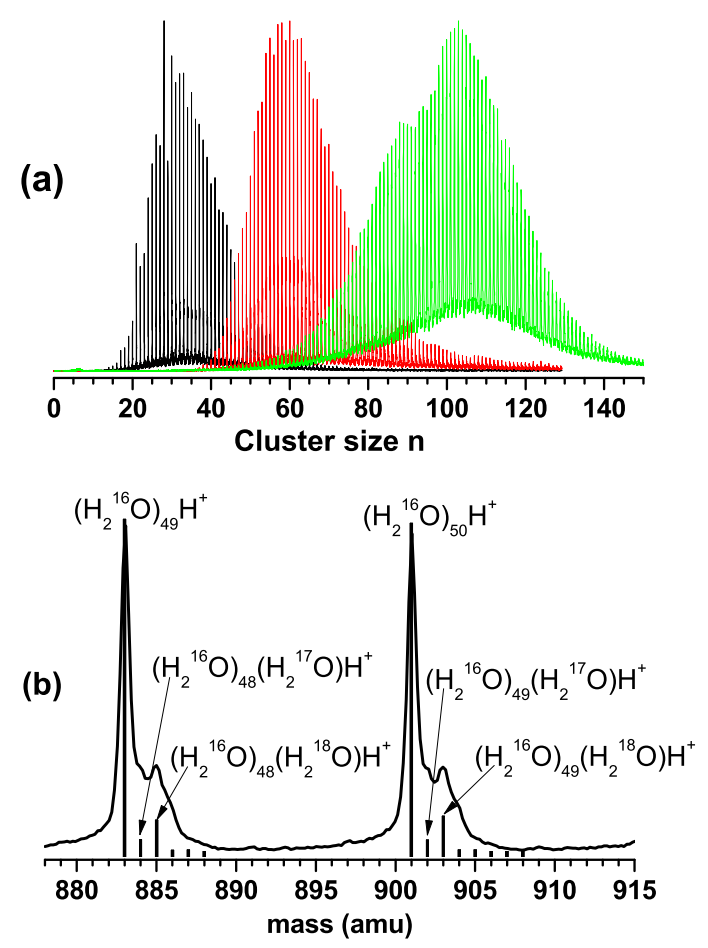

FIG. 2. (a) Mass spectra showing typical size distributions. As the amount of water is increased, from left to right, the size distribution shifts towards bigger sizes.

(b) Detailed structure of the peaks in the mass spectra. Clusters containing oxygen isotopes can be clearly distinguished. The vertical bars are the theoretical ratio of different isotopes.

left electrode of region (f): this way the slowest clusters are more reaccelerated and catch up with the fastest ones. Clusters are energy focused: they are all set to the same kinetic energy with a very good accuracy. Of course, the time at which $V_{f o c}$ is applied depends on the cluster mass under study. So this first step allows not only to energy focus but also contributes to the mass selection.

After being energy focussed the clusters enter the deceleration region (g). It consists in 12 electrodes to which increasing voltages are applied. When the clusters arrive at the end of the deceleration region (a constant field portion) the voltages are suddenly shut down. Then they fly freely at constant speed through the collision cell (h) until they reach the acceleration zone (i) where they are accelerated again towards the reflectron $(\mathrm{j})$ and detected by a microchannel plate detector $(\mathrm{k})$. Typical values for the voltages can be found 


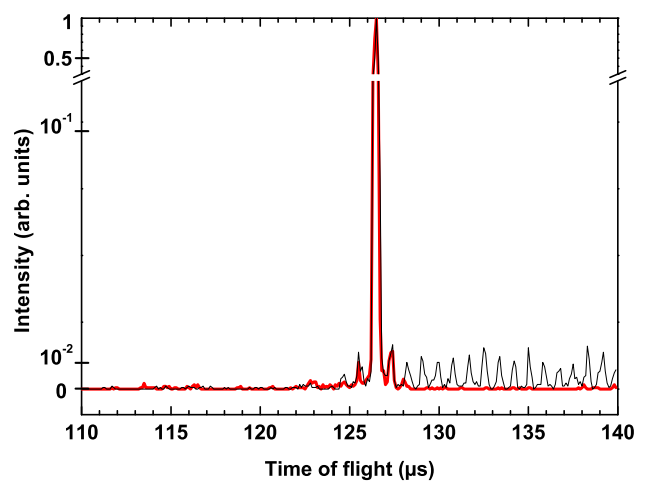

FIG. 3. Time-of-flight spectra for mass selected $\left(\mathrm{H}_{2} \mathrm{O}\right)_{70} \mathrm{H}^{+}$clusters slowed down to $33 \mathrm{eV}$. The thick (red) line using the mass filter.

in ref. 10 .

The combination of energy focussing followed by the deceleration allow us to slow down clusters down to $5 \mathrm{eV}$ kinetic energies in the laboratory frame with around $2 \mathrm{eV}$ dispersion.

The mass selection is the result of four successive operations. First only few masses around the one of interest are present in the energy focussing region (f) when $V_{\text {foc }}$ is applied. Then only the mass under study has been efficiently energy focussed and thus slowed down with the less losses. The decelerating voltage is shut down when the mass of interest reaches the constant field region of (g). Finally, only the selected mass with the desired kinetic energy will reach the reacceleration zone (i) at the right time.

Nevertheless, the mass selection is not perfect and small peaks corresponding to masses adjacent to the desired ones may appear in the time-of-flight (TOF) spectra. This is why an extra mass filtering step (e) has been added. It consists in a hollow cylinder, $30 \mathrm{~mm}$ long, with high transmission meshes on both sides. It is placed at the distance where the time spread for each cluster mass is the smallest. As soon as the desired mass enters the filter, a high voltage (2.5 $\mathrm{kV}$ typically) is applied to the cylinder so that higher masses cannot overcome the potential barrier. The high voltage is switched off just before the selected mass reaches the end of the cylinder. Masses smaller than the selected one come out of the filter while the high voltage is still on. They are thus reaccelerated and discarded as well.

Figure 3 shows an example of TOF spectrum for $\left(\mathrm{H}_{2} \mathrm{O}\right)_{70} \mathrm{H}^{+}$slowed down to $33 \mathrm{eV}$, with and without the mass filter. 


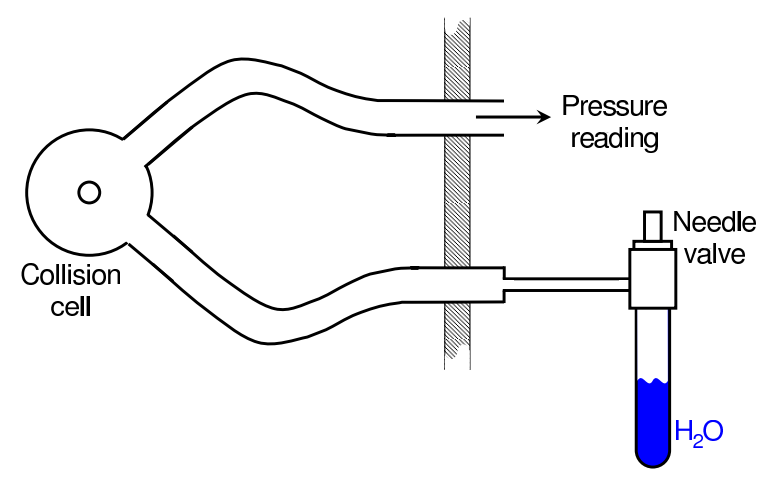

FIG. 4. Schematic of the collision cell.

Our experimental setup allows us to produce well controlled cluster-molecule collisions. Indeed we can perform collisions between mass selected, thermalized protonated water clusters with water molecules at a controlled kinetic energy, which can be set as low as $5 \mathrm{eV}$ in the laboratory frame. Furthermore, thanks to the excellent global transmission of about $50 \%$ one can study the whole size range of clusters produced by the source, so we can access the scaling law of attachment cross sections.

\section{Collision cell}

The collision cell (see fig. 4) is $50 \mathrm{~mm}$ long, $30 \mathrm{~mm}$ in diameter with $5 \mathrm{~mm}$ diameter entrance and exit holes. Water vapor is produced from a liquid water reservoir at ambient temperature. The amount of water vapor is precisely controlled by a needle valve. The water vapor is introduced in the cell through a $10 \mathrm{~mm}$ diameter pipe on the side of the cell. Another $10 \mathrm{~mm}$ diameter pipe connects the cell to two pressure gauges. The first gauge is an ionization gauge (Leybold Ionivac), the second one is a mechanical gauge (Leybold Ceravac CTR 91).

Measuring absolute cross sections requires a reliable pressure reading. Several constraints have guided us in the present configuration. The mechanical gauge does not give an absolute pressure reading. Indeed its zero has to be adjusted. On the other hand, it gives an accurate value of the relative pressure, whatever the gas used. Furthermore, due to its operation principle it is very sensitive to the vibrations generated by the helium closed cycle cryostat and gives rather noisy measurements. On the other hand the ionization gauge gives a stable 
signal but the pressure reading is very gas dependant. So we calibrate it with the help of the mechanical one when the helium cryostat is off. The calibration is done by recording both pressures as a function of the needle valve opening. The pressure measured by the ionization gauge is about 0.8 times the one measured by the mechanical gauge. During the signal acquisitions, both pressures are recorded continuously.

As will be detailed in section III, there is no need in having an absolute pressure measurement. We rather need a precise pressure variation measurement. The procedure described above insures us that the pressure variations we measure are accurate.

In order to measure reliable attachment cross sections one has also to wonder about the effective length of the cell: if pressure leaking outside the cell is important, then the clusters can collide with water molecules outside the cell, thus increasing the effective length of the cell. However, numerical simulations have shown that for collision cell similar to the one used in our experiment, leakage out of the cell could be neglected ${ }^{11}$. As a matter of fact, first, the pressure is significantly higher than the backgroung pressure outside the cell only over a very reduced range, of the order of the cell output holes diameter. Second, the pressure increase outside the cell is almost completely compensated by a pressure decrease inside the cell close to the apertures.

\section{STICKING CROSS SECTION MEASUREMENT}

Before they enter the collision cell, clusters have been thermalized, mass selected and they have a well defined translational kinetic energy $E_{k}$ in the laboratory frame. The collision cell contains a controlled pressure of water vapor. Within the cell, the cluster ions can undergo a number of collisions, and the resulting products are mass analyzed using the second TOF mass spectrometer.

Absolute attachment cross sections can be easily obtained from these mass spectra. Let us first consider figure 5 where are displayed several TOF spectra recorded for, from top to bottom, an increasing pressure in the cell. These spectra have been obtained by selecting $\left(\mathrm{H}_{2} \mathrm{O}\right)_{100} \mathrm{H}^{+}$and slow them down to $E_{k}=22 \mathrm{eV}$. The cluster temperature is $25 \mathrm{~K}$. The top spectrum illustrates our mass selection ability as no water vapor pressure is present in the cell: only one peak is present in the TOF spectrum corresponding to the $n=100$ cluster. As the pressure is increased one can observe more and more peaks in the TOF spectra 


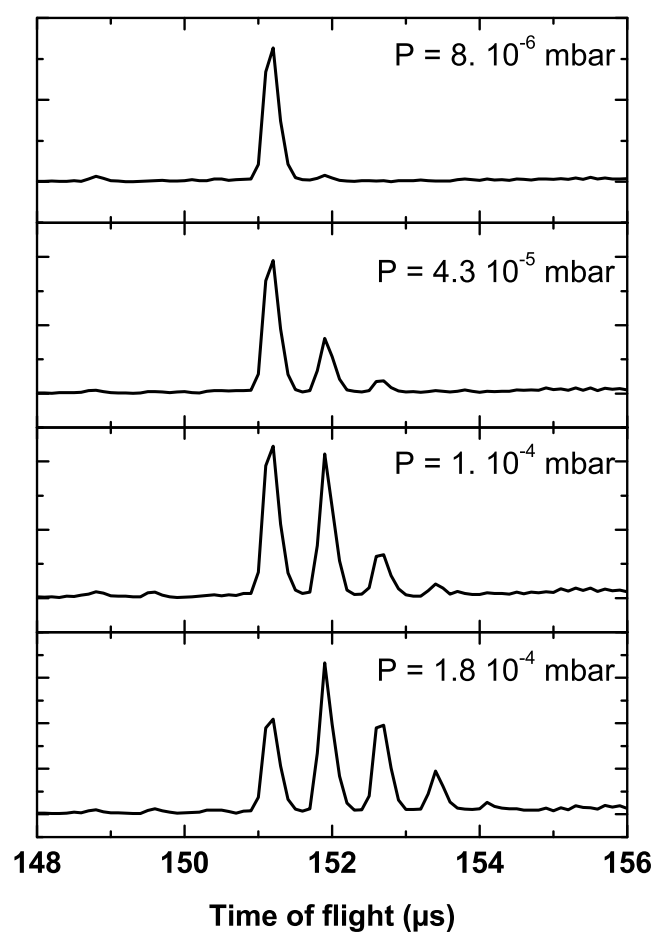

FIG. 5. Time-of-flight spectra of the products obtained after $\left(\mathrm{H}_{2} \mathrm{O}\right)_{100} \mathrm{H}^{+}$clusters have crossed the cell. From top to bottom, the number of attachments increases with the water vapor pressure in the cell. Clusters kinetic energy is $22 \mathrm{eV}$ and temperature is $25 \mathrm{~K}$.

corresponding to attachment events. In the bottom TOF spectrum of figure 5 one can see up to 4 water molecules added to the initial cluster.

The attachment cross section is easily obtained from the number of clusters that have undergone at least one sticking collision, and is given by ${ }^{12}$ :

$$
\sigma_{n}=-\frac{\ln I / I_{0}}{l \rho}\left\{\operatorname{erf}(\sqrt{a})+\frac{1}{2 a} \operatorname{erf}(\sqrt{a})+\frac{e^{-a}}{\sqrt{\pi a}}\right\}^{-1} .
$$

Here, $a=E_{k} /\left(n k_{B} T\right), l$ is the length of the cell, $\rho$ the molecular density inside the cell, $n$ the size of the incoming cluster, $T$ the temperature of the vapor, and erf is the error function. $I_{0}$ is the total number of incoming clusters whereas $I$ is the number of clusters that have undergone no sticking collisions. The first term on the right side of Eq. 1 comes from the standard Beer-Lambert law, while $F(a)=\operatorname{erf}(\sqrt{a})+\frac{1}{2 a} \operatorname{erf}(\sqrt{a})+\frac{e^{-a}}{\sqrt{\pi a}}$ accounts for 
the thermal distribution of molecules in the cell (see the appendix for details on how this formula is established). From mass spectra such as the ones presented in figure 5 one can easily determine $I$ and $I_{0}$ and calculate the attachment cross section using equation 1 . The main uncertainty in the cross section measurement then comes from the determination of the absolute gas density in the cell $\rho$. As mentioned in section II C it is rather difficult to measure accurately absolute pressures. On the other hand we have a reliable measure of the pressure variation. So in order to get rid of the uncertainty on the absolute pressure, we acquire the mass spectra as a function of the pressure and then plot $-\ln \left(I / I_{0}\right)$ as a function of $\rho l F(a)$. The slope of the obtained curve gives us directly the attachment cross section. Doing so, there is no need in knowing the absolute pressure. An example of such a curve is given in Figure 6, which has been obtained for $n=100$ and $E_{k}=22 \mathrm{eV}$. The perfect linearity of the curve insures that no evaporation takes place in the range of pressures explored. The complete elimination of evaporation effects in our experiment is an important issue which will be discussed in more details in the next section.

However measuring the cross sections in this way is quite time consuming. So, we use this method to obtain absolute cross sections for few sizes in the whole size range. Once this calibration is done, we acquire mass spectra at a fixed pressure in the collision cell for all sizes. This gives rapidly the size dependance of the attachment cross section but with a poorer accuracy on the absolute value. These data are then calibrated with the absolute measurements.

Both absolute and size dependant attachment cross sections have been measured several times, on different experimental runs and for different source conditions. From these different sets of measurement, we have estimated the error bars for the attachment cross section to be of the order of $\pm 10 \%$, which corresponds to the worse error observed for one given size. Figures 7 presents the attachment cross sections measured at $E_{k}=22 \mathrm{eV}$ as a function of the cluster size. The small squares in the figure corresponds to 12 independent size dependance measurements. The largest squares with error bars are the results of several absolute attachment cross section measurements averaged.

In the next sections we will present only the averaged measured cross sections without error bars in the figures for more clarity. 


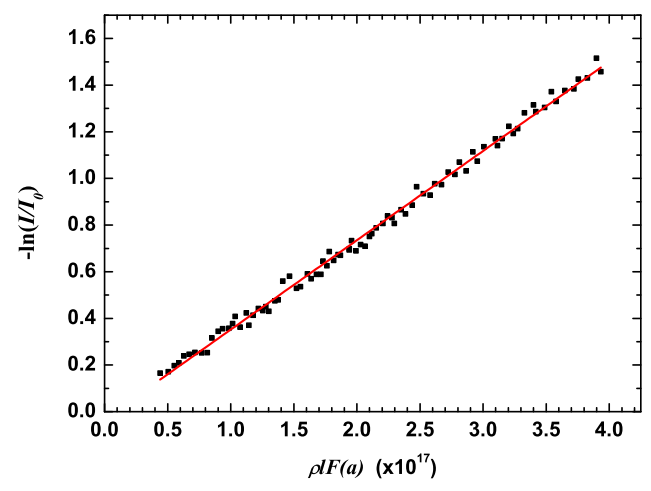

FIG. 6. Measurement of the attachment cross section of $\left(\mathrm{H}_{2} \mathrm{O}\right)_{100} \mathrm{H}^{+}$at a kinetic energy of $22 \mathrm{eV}$ in the laboratory frame. The attachment cross section is given by the slope of the curve $-\ln \left(I / I_{0}\right)$ plotted as a function of $\rho l F(a)$ (see text). Clusters temperature is $25 \mathrm{~K}$.

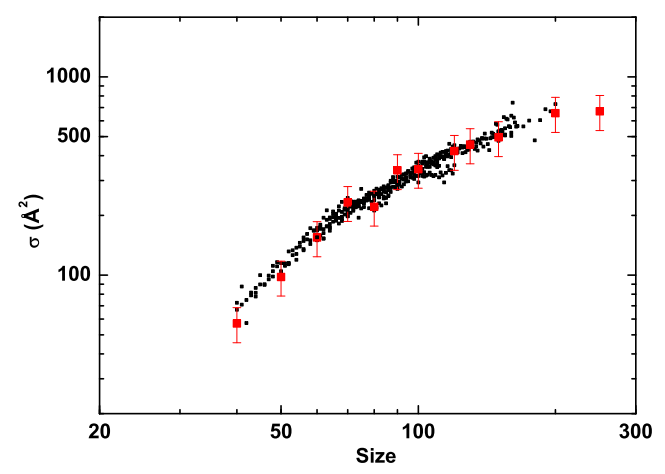

FIG. 7. Attachment cross sections of water molecules onto protonated water clusters as a function of their size. The experiments were performed for a kinetic energy of $22 \mathrm{eV}$ in the laboratory frame. The small squares correspond to rapid acquisitions of the size dependance whereas the large squares with error bars are absolute attachment cross sections measurements (see text). Clusters temperature is $25 \mathrm{~K}$.

\section{EXPERIMENTAL RESULTS AND DISCUSSION}

\section{A. Temperature dependence of attachment cross sections}

In order to measure attachment cross sections, one has to pay particular attention to the possible evaporation of the clusters on the time scale of the experiment. Indeed, after the 
attachment of a water molecule, the initial internal energy $E_{i}$ of the clusters of size $n$ will become:

$$
E_{f}=E_{i}+D+E_{c}
$$

where $D$ is the dissociation energy of size $n+1$ and $E_{c}$ is the mean value of the collision energy in the center of mass frame:

$$
E_{c}=\frac{E_{k}}{n+1}+\frac{3 k_{B} T}{2} \frac{n}{n+1} .
$$

In this equation, $k_{B}$ is the Boltzmann constant, $T$ the temperature of the vapor and $E_{k}$ the cluster kinetic energy in the laboratory frame.

If $E_{f}$ becomes too large, the lifetime of the clusters might become shorter than the experiment timescale. One would then detect clusters at size $n$ even if they have undergone an attachment. Evaporation can therefore complicate the interpretation of the results as it is the case in previous experiments ${ }^{13}$. This is why we make sure that no evaporation takes place during the time of our experiments.

Let us first make a rough estimate of the internal energy reached in our experiment. We consider for instance clusters of size $n=50$, slowed down at $33 \mathrm{eV}$ in the laboratory frame, which is about the highest collision energy employed in the experiments presented in this paper. This collision energy is about $E_{c}=0.68 \pm 0.04 \mathrm{eV}$.

The dissociation energies for protonated water clusters are about $0.45 \mathrm{eV}^{14}$. The total energy deposited upon attachment in the clusters is thus about $1.1 \mathrm{eV}$.

The energy required to reach evaporation can be estimated from recent calorimetric measurements made for negatively charged water clusters ${ }^{15}$. Even if the experiment in ref. 15 has been performed for negatively charged clusters, preliminary studies in our group show very similar behavior for positively charged protonated clusters. From this work, one can deduce the initial internal energy $E_{i}$ at our working temperature $T_{t h}=25 \mathrm{~K}$ and the internal energy $E_{\text {evap }}$ required to reach evaporation. We find $E_{i}\left(T_{t h}=25 \mathrm{~K}\right)=0.05 \mathrm{eV}$ and $E_{\text {evap }}=1.25 \mathrm{eV}$.

In the above example the final internal energy is thus of the order of the energy required to get evaporation. Of course, the experimental conditions in ref. 15 are different from ours (negatively charged clusters instead of protonated ones, timescales of the experiment). Furthermore the case considered here is somewhat extreme: the energy deposited is lower 
TABLE I. Temperature $T_{\text {drop }}$ at which the attachment cross section starts to drop (see fig. 8) for different cluster sizes and kinetic energies in the laboratory frame.

\begin{tabular}{lll}
\hline \hline Size $n$ & $E_{k}(\mathrm{eV})$ & $T_{\text {drop }}(\mathrm{K})$ \\
60 & 22 & $125 \pm 5$ \\
60 & 33 & $110 \pm 10$ \\
50 & 33 & $85 \pm 5$ \\
45 & 33 & $70 \pm 10$ \\
\hline \hline
\end{tabular}

for lower collision energy and bigger sizes. Nevertheless, even for this extreme case we show below by a more careful experimental analysis that there is no evaporation as far as the initial temperature is kept low enough.

The absence of evaporation has been verified by measuring the variation of the experimental cross section as a function of the initial temperature of the clusters. This measurement has been done for a few sizes and collision energies. The experimental results for $\left(\mathrm{H}_{2} \mathrm{O}\right)_{50} \mathrm{H}^{+}$ at $33 \mathrm{eV}$ are presented in figure 8(a). When the internal energy is high enough to induce evaporation before the detector, the measured attachment cross section drops abruptly. This drop occurs here at a temperature $T_{\text {drop }}$ of $85 \pm 5 \mathrm{~K}$. In figure $8(\mathrm{~b})$ we present the temperature $T_{\text {drop }}$ as a function of the collision energy in the center of mass frame (the corresponding sizes and kinetic energy in the laboratory frame can be found in table I). As expected the temperature $T_{d r o p}$ decreases as the collision energy increases. A simple estimate of $T_{d r o p}$ can be made by considering that the final energy $E_{f}$ reached in the different cases listed in table I corresponds to the same evaporation rate. If we consider the rotational and vibrational degree of freedom of the water molecules to be freezed in the cluster at the considered temperatures, one can write the final energy $E_{f}$ as:

$$
E_{f}=(3 n-6) k_{B} T_{f}=(3 n-6) k_{B} T_{\text {drop }}+D+E_{c}
$$

We then consider the evaporation rate $k_{\text {evap }}$ to be constant:

$$
k_{\text {evap }}=\alpha e^{-D / k_{B} T_{f}}
$$

In equation 5, $\alpha$ is a prefactor which depends on the model employed to derive the evaporation rate. It is not expected to vary substantially over the size range considered here and 
will be considered constant in the following. Defining the constant $C=\ln \left(\alpha / k_{\text {evap }}\right)$, the following expression is obtained for $T_{d r o p}$ :

$$
T_{\text {drop }}=\frac{D}{C k_{B}}-\frac{D+E_{c}}{k_{B}(3 n-6)}
$$

We are left with a single parameter to reproduce the experimental data, namely the constant $C$. The calculated values of $T_{\text {drop }}$ with $D=0.45 \mathrm{eV}^{14}$ and $C=29$ (plotted as a line in figure $8(\mathrm{~b})$ ) are in good agreement with the experimental points. It supports our assumption about the drop in cross section with temperature being due to thermal evaporation.

Since all attachment cross section measurements are performed at $25 \mathrm{~K}$, we can safely exclude any effect of evaporation on our measurements.

\section{B. Size and collision energy dependence of the attachment cross section}

We present in figure 9 the cross sections for the attachment reactions $\left(\mathrm{H}_{2} \mathrm{O}\right)_{n} \mathrm{H}^{+}+\mathrm{H}_{2} \mathrm{O} \rightarrow$ $\left(\mathrm{H}_{2} \mathrm{O}\right)_{n+1} \mathrm{H}^{+}$measured at five kinetic energies in the laboratory frame, $E_{k}=6,10,18,22$ and $33 \mathrm{eV}$. The corresponding center of mass collision energy range is $0.1-0.7 \mathrm{eV}$.

On the same figure is also plotted the hard sphere cross section $\sigma_{\text {geo }}$ (dotted line in Fig. 9). It is calculated as $\sigma_{\text {geo }}=\pi\left(R_{\text {cluster }}+r_{\text {molecule }}\right)^{2}$, with $R_{\text {cluster }}=r_{\text {molecule }} n^{1 / 3}$. We used $r_{\text {molecule }}=2.17 \AA$. The choice of this value will be discussed in section IV D (note that the molecular radius deduced from the density of bulk ice is $1.98 \AA$ ).

The figure 10 displays the attachment cross section measured for $\left(\mathrm{H}_{2} \mathrm{O}\right)_{70} \mathrm{H}^{+}$as a function of the collision energy in the center of mass frame. The cross section decreases smoothly with the collision energy.

The attachment cross sections decrease smoothly as the size decreases. For a given size, it also decreases as the collision energy increases (see figure 10). These two features are linked. Indeed, Eq.3 shows that at constant laboratory kinetic energy, the collision energy in the center of mass frame increases as the size decreases.

The measured cross sections for the five different kinetic energies converge towards a unique curve compatible with the hard sphere cross section approximation for sizes above $n \sim 100$.

The main surprise in these results comes from the fact that we find attachment cross 

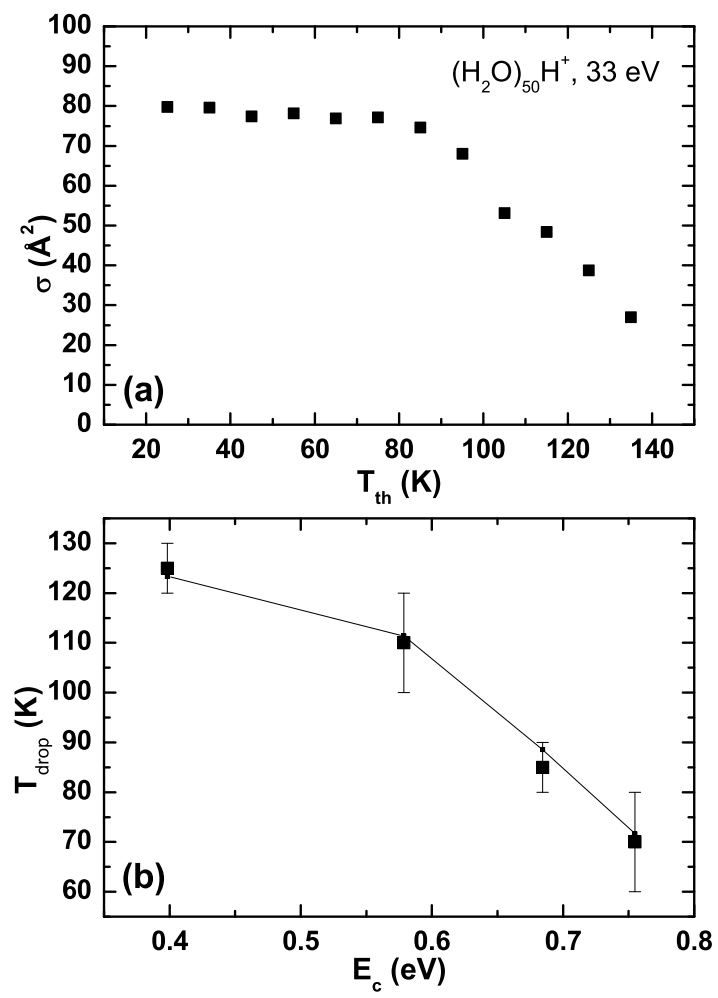

FIG. 8. (a) Sticking cross section of $\left(\mathrm{H}_{2} \mathrm{O}\right)_{50} \mathrm{H}^{+}$as a function of the thermalization temperature $T_{t h}$.

(b) Temperatures at which the attachment cross section starts to drop as a function of the collision energy. The squares correspond to the experimental data points presented in table I. The lineconnected small squares are calculated temperatures (see text).

sections always lower than geometrical cross sections for all the sizes and collision energies explored. For instance, the lowest collision energy in figure 10 is about $0.1 \mathrm{eV}$, which is only about twice the thermal collision energy. Even at this low collision energy we recover only $3 / 4^{\text {th }}$ of the geometrical cross section.

We have already ruled out in ref. 8 different mechanisms that could explain these results. Namely, we considered exchange reactions where the impinging molecule ejects one molecule from the cluster and takes its place and showed that, although present, this mechanism is not sufficient to explain our results. We also showed that charge exchange during the collision is not energetically allowed. Finally, as shown in section IV A evaporation is absent in our 


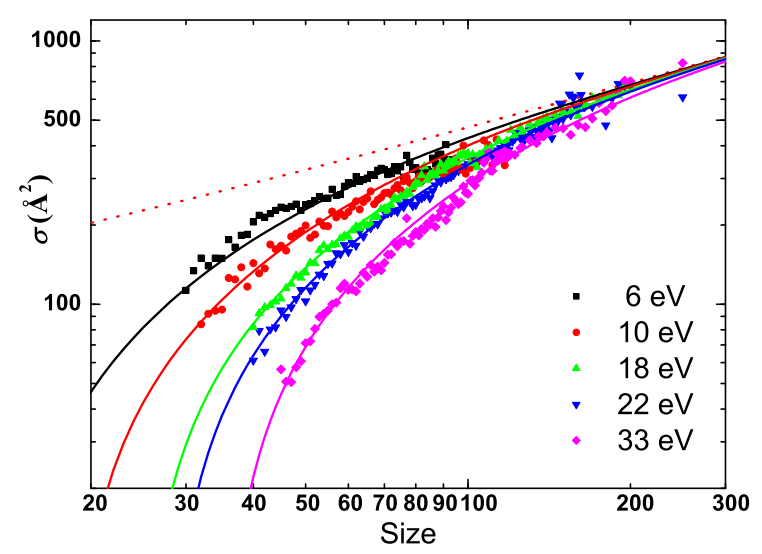

FIG. 9. Log-Log plot of the water clusters-water molecule attachment cross section as a function of cluster size for five different kinetic energies in the laboratory frame. Experimental results are compared to the hard sphere model (dotted line). The full lines through the data points are the prediction of the model presented in section IV D.

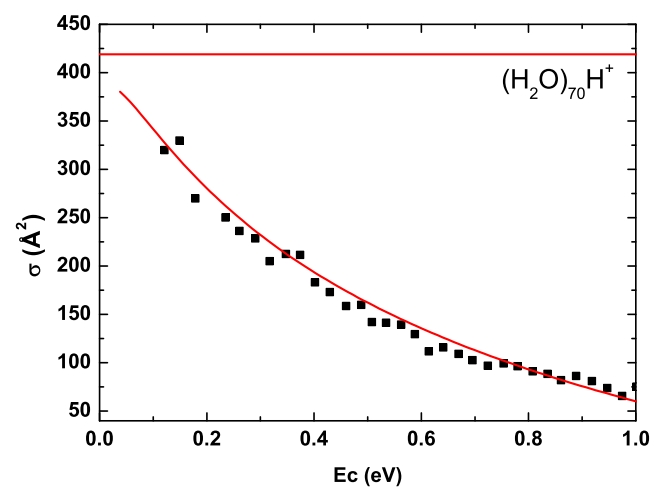

FIG. 10. Experimental attachment cross section (black squares) of $\left(\mathrm{H}_{2} \mathrm{O}\right)_{70} \mathrm{H}^{+}$as a function of collision energy in the center of mass frame. The horizontal line at the top of the figure is the geometrical cross section. The full line through the data points is the prediction of the model presented in section IV D.

experiment.

Before we rationalize our results with the help of a simple model (see section IV D), let us compare our results with the predictions of simple Langevin models. 


\section{Langevin cross sections}

Deviation from geometrical cross sections at small sizes has been predicted for neutral reagents ${ }^{16}$ and experimentally observed in the attachment of sodium atoms onto positively charged sodium clusters ${ }^{7}$. Sticking cross sections of sodium atoms onto sodium clusters are significantly higher than hard sphere cross sections at low collision energy, which is qualitatively understood in terms of a model based on a charge-induced dipole interaction ${ }^{7}$.

Water clusters not only interact with water molecules through charge-induced dipole interaction, but also through the charge-permanent dipole interaction, both of which are attractive forces. Furthermore, the attractive short range interaction is expected to result in an enhancement of the attachment cross section ${ }^{17}$, especially at small size and low collision energy $^{13,18}$.

Collision cross sections can be estimated with the help of the average dipole orientation (ADO) theory in the frame of a Langevin approach ${ }^{19}$. Let us consider that the interaction between the charged cluster and the neutral water molecule is given by (in atomic units):

$$
V(r)=-\frac{\alpha}{2 r^{4}}-\frac{C_{l} \mu_{D}}{r^{2}}+\frac{E_{c} b^{2}}{r^{2}}
$$

The first term represents the charge-induced dipole interaction, the second term is the charge-permanent dipole interaction and the third term is the centrifugal barrier. $r$ is the distance between the center of the cluster and the molecule, $b$ is the impact parameter. The polarisability of water is $\alpha=9.93$ a.u. ${ }^{20}$ and the dipole moment of water $\mu_{D}$ equals 0.73 a.u. ${ }^{21} . \quad C_{l}$ is the dipole locking constant introduced by $\mathrm{Su}$ et al ${ }^{19}$ in $\mathrm{ADO}$ theory. According to ref. 19 the dipole locking constant is here $C_{l}=0.215$. The classical expression for the collision cross section in the ADO theory is:

$$
\sigma_{A D O}=\pi b_{A D O}^{2}
$$

with

$$
b_{A D O}^{2}=\frac{C_{l} \mu_{D}}{E_{c}}+\sqrt{\frac{2 \alpha}{E_{c}}}
$$




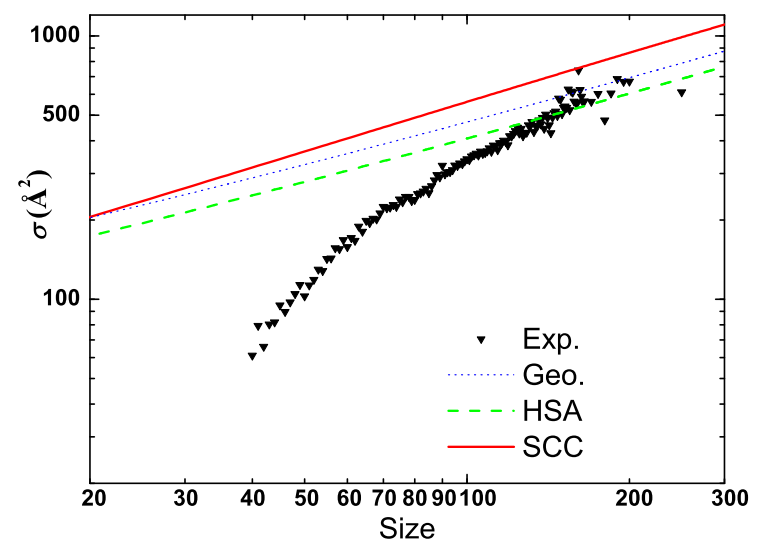

FIG. 11. Log-Log plot of the attachment cross section as a function of cluster size for $22 \mathrm{eV}$ kinetic energy in the laboratory frame. Experimental results (triangles) are compared to two Langevin models: HSA (dashed line) and SCC (full line). The hard sphere model obtained with $r_{\text {molecule }}=2.17 \AA$ is plotted as a dotted line.

This crude model does not take into account the finite size of the cluster. Size effects are introduced using the models developed by Kummerlöwe et $a l^{22}$. Two models are considered, the Hard Sphere Average dipole orientation (HSA) and the Surface Charge Capture (SCC) model. In the HSA model, the charge is considered to be motionless at the center of the cluster. In the SCC model the charge is mobile and located at the surface of the cluster (the distance between the charge and the neutral partner is the smallest geometrically allowed).

The expressions for attachment cross sections are the following:

$$
\begin{aligned}
\sigma_{H S A} & =\sigma_{A D O}, \text { if } E_{c} \leq E^{*} \\
& =\sigma_{g e o}+\pi\left(\frac{C_{l} \mu_{D}}{E_{c}}+\frac{\alpha}{2 D^{2} E_{c}}\right), \text { if } E_{c}>E^{*}
\end{aligned}
$$

with $D=R_{\text {cluster }}+r_{\text {molecule }}$ and $E^{*}=\frac{\alpha}{2 D^{4}}$ is the collision energy for which $\sigma_{A D O}$ becomes smaller than $\sigma_{g e o}$.

$$
\sigma_{S C C}=\pi\left(R_{\text {cluster }}+b_{A D O}\right)^{2}
$$

We have plotted in figure 11 the HSA and SCC cross sections, using $r_{\text {molecule }}=1.98 \AA$ as deduced from bulk ice density. We compare the HSA and SCC cross sections to the 
experimental results at $22 \mathrm{eV}$ kinetic energy in the laboratory frame. Experimental cross sections converge asymptotically right in between the predictions given by these two models.

Equations 10 and 11 give lower and upper limits for the attachment cross sections, respectively. They run almost parallel to the geometric cross section for the sizes and kinetic energies considered in this work. Since these electrostatic models do not depart significantly from hard sphere cross sections and do not account for the experimental drop at small sizes, we will consider the deviation from the hard sphere cross section as a relevant quantity.

\section{Dynamical effects}

Our experimental measurements of attachment cross sections of water molecules onto water clusters show that, as the collision energy increases, these cross sections become smaller than geometric cross sections. Reactive channels other than attachment and statistical thermal decay have been eliminated as a possible cause of this behavior. The only way to explain this reduction of the attachment efficiency at high collision energy is thus to consider that even in some head-on collisions the impinging molecule is merely deflected without undergoing attachment. A reason for this may be that the collision energy has not been absorbed during the collision. We rationalized this idea with the help of a simple model. The attachment of a water molecule to the cluster requires the formation of a collision complex with a sufficiently long lifetime to allow the collision energy to be redistributed among the internal degree of freedom of the complex. On the other hand, if the collision duration $\tau_{c}$ is short compared to the relevant vibrational period $\tau_{v}$ for energy redistribution, the impinging molecule bounces off the cluster. This can also be stated by introducing an adiabaticity parameter, $\xi=\tau_{c} / \tau_{v}$, in order to separate adiabatic from sudden regimes ${ }^{23}$. One has therefore to quantify the amount of inelastic collisions that does not lead to attachment. This quantity can be written as:

$$
P_{\text {inelastic }}=\left(\sigma_{\text {geo }}-\sigma_{\text {exp }}\right) / \sigma_{\text {geo }}
$$

where $\sigma_{\text {exp }}$ is the measured cross section and $\sigma_{g e o}$ the geometric cross section.

The duration of the collision can be estimated by considering the time required for an impinging molecule to cover a distance of the order of the cluster diameter:

$$
\tau_{c}=2 n^{1 / 3} r_{\text {molecule }}\left(2 E_{k} /(n m)+3 k_{B} T / m\right)^{-1 / 2}
$$


Here $m$ and $r_{\text {molecule }}$ are the mass and radius of a single water molecule, respectively. In our experiment $\tau_{c}$ ranges from 0.5 ps to $2.4 \mathrm{ps}$.

When $P_{\text {inelastic }}$ is plotted as a function of the collision duration $\tau_{c}$, all the experimental data points presented in figure 9 fall on the same curve (see figure 3 of ref. 8) which can be simply fitted by the function $e^{-\left(\tau_{c}-\tau_{0}\right) / \tau_{v}}$. The attachment cross section can then be expressed as:

$$
\sigma=\sigma_{g e o}\left(1-e^{-\left(\tau_{c}-\tau_{0}\right) / \tau_{v}}\right)
$$

In order to reproduce the experimental results with this expression several parameters have to be determined. Namely $\tau_{v}, \tau_{0}$ and the molecular radius $r_{\text {molecule }}$ which enters in the expression of the geometric cross section $\sigma_{\text {geo }}=\pi\left(R_{\text {cluster }}+r_{\text {molecule }}\right)^{2}$ and of the collision duration (Eq. 13).

These parameters are deduced from a fitting procedure on the experimental data. The results of the fit obtained using the expression given in eq. 14 are plotted as continuous lines in figure 9. This simple model is able to reproduce satisfactorily the experimental results. The best fit to the experimental data is obtained for $\tau_{0}=0.45 \mathrm{ps}, r_{\text {molecule }}=2.17 \AA$ and $\tau_{v}=0.62 \mathrm{ps}$. The model also nicely reproduces the variation of the cross sections with collision energy (see fig. 10).

The value of $\tau_{v}$ deduced from the fit corresponds to an energy of $6.7 \mathrm{meV}$. Brudermann et $a l^{9,24}$ have shown that the main low-energy surface vibrational mode excited by collisions of rare gas atoms onto water clusters is the $\mathrm{O} \cdot \cdot \mathrm{O} \cdot \mathrm{O}$ bending mode. This mode has an energy of about $5 \mathrm{meV}$. Its energy increases slightly with the size of the clusters and approaches asymptotically the result for the dispersionless surface phonons of ice ${ }^{25}$ : it goes from $4.3 \mathrm{meV}$ for $\mathrm{n}=22$ to $5.5 \mathrm{meV}$ for $\mathrm{n}=194$. The value deduced from our experiment is compatible with the $\mathrm{O} \cdot \mathrm{O} \cdot \mathrm{O}$ bending mode energy. Furthermore, if one fixes $\tau_{v}$ at a value of 0.8 ps (which corresponds to $5 \mathrm{meV}$ ), our data are still very satisfactorily reproduced with $r_{\text {molecule }}=2.25 \AA$ and $\tau_{0}=0.4 \mathrm{ps}$.

The value of the molecular radius deduced from the fit is slightly higher than that one would deduce from the density of bulk ice. This discrepancy can originate from several effects. First, considering clusters as spheres is of course a crude approximation. Second, the structure of clusters is not the same as in the bulk: there is no evidence that the density is the same. Third, as shown in section IV C, it is still possible that the cross section is 
slightly enhanced due to the attractive interaction between the cluster and the molecule. Finally, the difference between the fitted value and the one deduced from ice density is only about $10 \%$. This is within the error bar for the absolute value of the measured cross sections.

\section{E. Comparison with heavy water clusters}

We also performed experiments with deuterated water (Aldrich, 99.99\%). We introduced $D_{2} \mathrm{O}$ both in the source and the collision cell to measure the attachment cross section of the reaction $\left(D_{2} O\right)_{n} D^{+}+D_{2} O \rightarrow\left(D_{2} O\right)_{n+1} D^{+}$. The experiment has been performed at $E_{k}=10$ and $22 \mathrm{eV}$ and the attachment cross sections measured as a function of the cluster size. We compare in figure 12 the cross sections for $\left(\mathrm{H}_{2} \mathrm{O}\right)_{n} H^{+}$and $\left(\mathrm{D}_{2} \mathrm{O}\right)_{n} \mathrm{D}^{+}$. We find the same general evolution for heavy water clusters as for normal water clusters. Namely, the cross section decreases with increasing kinetic energy and/or decreasing size. More interesting is the comparison with the $\left(\mathrm{H}_{2} \mathrm{O}\right)_{n} H^{+}$clusters. At both collision energies, we find significantly higher cross sections for heavy water clusters than for light water clusters, especially for small sizes and high kinetic energies.

Even by taking into account the uncertainty in the absolute value of the cross section, we are quite confident in the relative values. That is, systematic errors in the determination of $\left(\mathrm{H}_{2} \mathrm{O}\right)_{n} \mathrm{H}^{+}$and $\left(\mathrm{D}_{2} \mathrm{O}\right)_{n} \mathrm{D}^{+}$attachment cross sections are the same.

The fitting procedure described in section IV B has been applied to the heavy water

clusters. The intermolecular vibrational mode energy has been taken as $6.7 \mathrm{meV} \times \sqrt{\frac{18}{20}}$ to account for the mass difference between $\mathrm{H}_{2} \mathrm{O}$ and $\mathrm{D}_{2} \mathrm{O}$. We can also satisfactorily reproduce the experimental results.

The molecular radius deduced from the fit is slightly higher than for light water, with a value of $r_{D_{2} O}=2.25 \AA$. This is not easily explained since from the heavy water bulk ice density, the molecular radius is almost the same as the one for $H_{2} O$, namely $1.98 \AA$. The difference in the molecular radius deduced for $\mathrm{D}_{2} \mathrm{O}$ and $\mathrm{H}_{2} \mathrm{O}$ data is only about $3.6 \%$. We can hardly claim that we achieve such a precision in our measurements. Nevertheless, this difference between light and heavy water is within the range of structural changes observed by Soper and Benmore by X-ray diffraction ${ }^{26}$. In particular they found that the hydrogen bond length in light water is $\sim 4 \%$ shorter than in heavy water.

Nevertheless, for a given size and kinetic energy in the laboratory frame, the attachment 


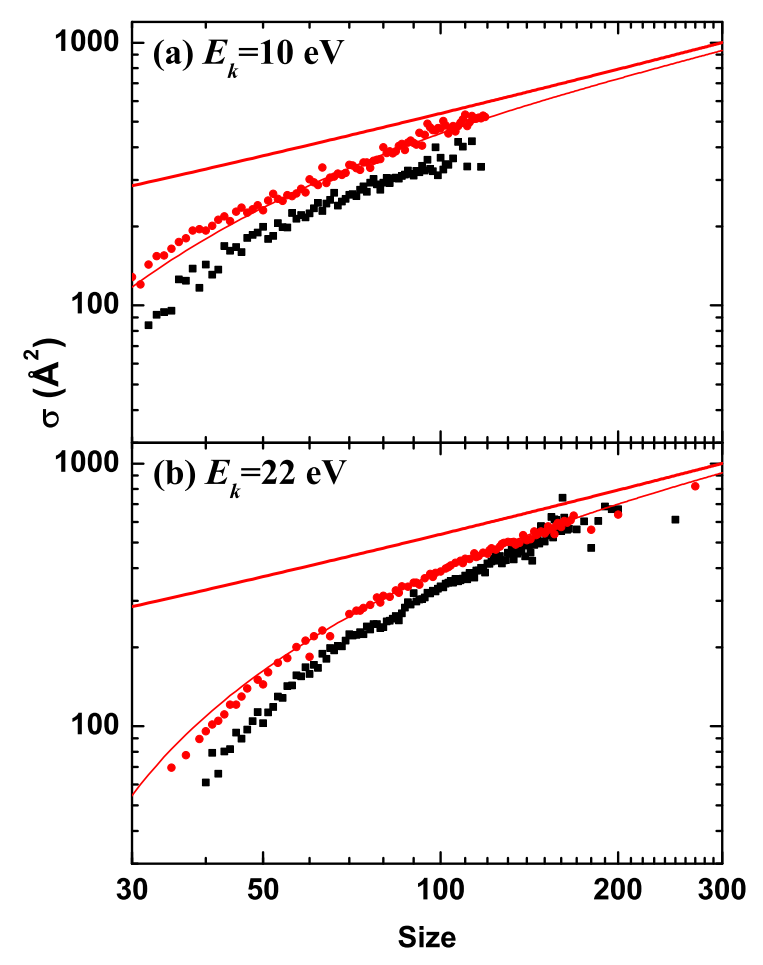

FIG. 12. Comparison of the attachment cross sections for $\left(\mathrm{H}_{2} \mathrm{O}\right)_{n} \mathrm{H}^{+}$(squares) and $\left(\mathrm{D}_{2} \mathrm{O}\right)_{n} \mathrm{D}^{+}$ (circles) clusters as a function of the size $n$. The top panel corresponds to $E_{k}=10 \mathrm{eV}$ whereas the bottom one corresponds to $E_{k}=22 \mathrm{eV}$. The full thin lines correspond to the model described in section IV D. The geometrical cross section is plotted as full thick lines.

cross section is clearly higher for heavy water clusters than for light water ones. This difference can not be attributed to heavy water clusters being "bigger" than the light ones. More interestingly, the experiments with heavy water clusters supports the hypothesis of inelastic collisions due to dynamical effect. Indeed, once plotted as a function of the cluster mass rather than the size, or equivalently as a function of the collision duration, the cross sections for heavy and light water clusters get very close to each other (see figure 13). This is particularly true for $E_{k}=22 \mathrm{eV}$ (bottom part of figure 13) where the attachment cross sections almost perfectly overlap. According to our model, this difference in cross section can be attributed to the longer collision time for heavy water clusters. 


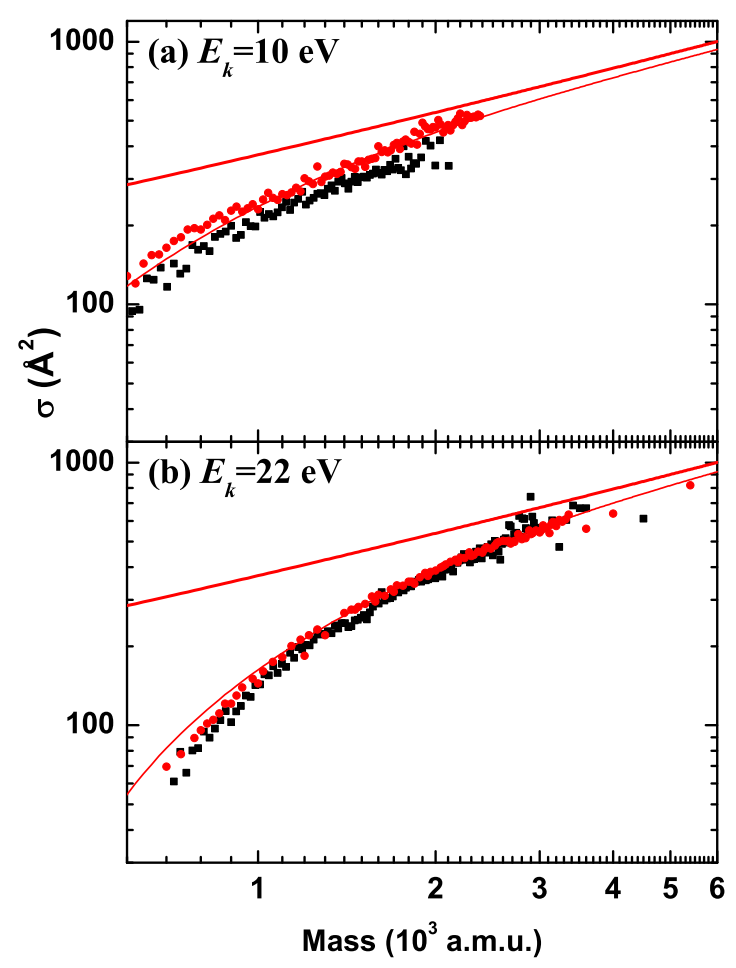

FIG. 13. Comparison of the attachment cross sections for $\left(\mathrm{H}_{2} \mathrm{O}\right)_{n} \mathrm{H}^{+}$(squares) and $\left(\mathrm{D}_{2} \mathrm{O}\right)_{n} \mathrm{D}^{+}$ (circles) clusters as a function of the cluster mass. The top panel corresponds to $E_{k}=10 \mathrm{eV}$ whereas the bottom one corresponds to $E_{k}=22 \mathrm{eV}$. The full thin lines correspond to the model described in section IV D. The geometrical cross section is plotted as full thick lines.

\section{CONCLUSION}

We have measured absolute attachment cross section of water molecules onto protonated water cluster. We are able to completely rule out the role of evaporation in our experiment. We have found that, within our experimental conditions, the cross section never exceeds the geometrical cross section. Our results are well explained by dynamical effects: if the duration of the collision is too short, there is not enough time for the cluster to transfer the collision energy into the internal vibrational modes. Comparison with deuterated water clusters further support this interpretation. Based on this assumption we built a simple model which satisfactorily reproduce our experimental results (size and energy dependance).

This work suggests that dynamical effects should be given more careful consideration 
in the study of the growth of microscopic systems. For instance, standard equilibrium thermodynamical approaches, based on the assumption of permanent statistical distribution of energy between all degrees of freedom, do not take this kind of dynamical effect into account.

\section{Appendix: Calculation of the cross section}

We consider clusters with velocity $v_{\text {cluster }}$ crossing a water vapor at temperature $T$. The velocities $v$ of the molecules in the vapor follow the Boltzman distribution $f(v)$ :

$$
f(v)=\left(\frac{m}{2 \pi k_{B} T}\right)^{3 / 2} e^{-\frac{1}{2} m v^{2} / k_{B} T}
$$

where $m$ is the water molecule mass, $k_{B}$ the Boltzman constant and $T$ the vapor temperature.

Let us consider $I_{0}$, the number of parent clusters before the cell and $I$ the number of parent clusters after the cell. During a time $d t$, the variation of the number of parent clusters is given by:

$$
d I=-\rho \sigma \int d^{3} v f(v) v_{r e l} I d t
$$

where $\rho$ is the density in the cell and $\sigma$ the attachment cross section. $v_{r e l}$ is the relative velocity of the clusters to the vapor molecules and is given by:

$$
v_{\text {rel }}=\sqrt{v_{\text {cluster }}^{2}+v^{2}-2 v v_{\text {cluster }} \cos \theta} \text {. }
$$

And the parent cluster number at the output of the cell is simply:

$$
I=I_{0} e^{-\rho \sigma \int d^{3} v v_{\text {rel }} t_{\text {cross }}}
$$

where $t_{\text {cross }}$ is the time it requires for the clusters to cross the cell. This time is simply $t_{\text {cross }}=l / v_{\text {cluster }}$ where $l$ is the cell length and $v_{\text {cluster }}$ the cluster velocity.

If one neglects the velocity distribution, one can get the attachment cross section as:

$$
\sigma=-\frac{\ln \left(I / I_{0}\right)}{\rho l} \frac{v_{\text {cluster }}}{\left\langle v_{\text {rel }}\right\rangle} \text {. }
$$

where $\left\langle v_{\text {rel }}\right\rangle$ is the average value. Eq. A.5 can be rewritten as:

$$
\sigma=-\frac{\ln \left(I / I_{0}\right)}{\rho l}\left(1+\frac{3 n k_{B} T}{2 E_{k}}\right)^{-1 / 2}
$$


On the other hand, in order to take into account the velocity distribution of the molecules in the vapor, the following integral has to be evaluated:

$$
F=\left(\frac{m}{2 \pi k_{B} T}\right)^{3 / 2} \int_{-1}^{1} d(\cos \theta) \int_{0}^{\infty} v^{2} d v \sqrt{v_{\text {cluster }}^{2}+v^{2}-2 v v_{\text {cluster }} \cos \theta} e^{-\frac{1}{2} m v^{2} / k_{B} T}
$$

which gives:

$$
F=\operatorname{erf}(\sqrt{a})+\frac{1}{2 a} \operatorname{erf}(\sqrt{a})+\frac{e^{-a}}{\sqrt{\pi a}}
$$

where $a=E_{k} /\left(n k_{B} T\right)$ and $\operatorname{erf}(x)=\frac{2}{\sqrt{\pi}} \int_{0}^{x} e^{-t^{2}} d t$ is the error function. The expression for the attachment cross section now reads:

$$
\sigma=-\frac{\ln \left(I / I_{0}\right)}{\rho l}\left(\operatorname{erf}(\sqrt{a})+\frac{1}{2 a} \operatorname{erf}(\sqrt{a})+\frac{e^{-a}}{\sqrt{\pi a}}\right)^{-1}
$$

\section{ACKNOWLEDGMENTS}

We gratefully acknowledge J. Vigué for fruitful discussions.

\section{REFERENCES}

${ }^{1}$ R. Becker and W. Döring, Annalen der Physik 416, 719 (1935).

${ }^{2}$ I. Ford, Phys. Rev. E 56, 5615 (1997).

${ }^{3}$ D. W. Oxtoby, J. Phys.: Condens. Matter 4, 7627 (1992).

${ }^{4}$ J. Feder, K. Russel, J. Lothe, and G. Pound, Adv. Phys. 15, 111 (1966).

${ }^{5}$ M. Anisimov, Russ. Chem. Rev. 72, 591 (2003).

${ }^{6}$ J.-M. L'Hermite, Phys. Rev. E 80, 051602 (2009).

${ }^{7}$ F. Chirot, P. Labastie, S. Zamith, and J.-M. L'Hermite, Phys. Rev. Lett. 99, 193401 (2007).

${ }^{8}$ S. Zamith, P. Feiden, P. Labastie, and J.-M. L'Hermite, Phys. Rev. Lett. 104, 103401 (2010).

${ }^{9}$ J. Brudermann, P. Lohbrandt, U. Buck, and V. Buch, Phys. Rev. Lett. 80, 2821 (1998).

${ }^{10}$ F. Chirot, S. Zamith, P. Labastie, and J.-M. L'Hermite, Rev. Sci. Instrum. 77, 063108 (2006). 
${ }^{11}$ B. P. Mathur, J. E. Field, and S. O. Colgate, Phys. Rev. A 11, 830 (1975).

${ }^{12}$ Note that in ref. ${ }^{7}$ and $^{8}$ the exponent ${ }^{-1}$ is missing in the formula for the cross section.

${ }^{13}$ Y. Okada, S. Yamaguchi, Y. Kawai, T. Orii, and K. Takeuchi, Chem. Phys. 294, 37 (2003).

${ }^{14}$ K. Hansen, P. U. Andersson, and E. Uggerud, J. Chem. Phys. 131, 124303 (2009).

${ }^{15}$ C. Hock, M. Schmidt, R. Kuhnen, C. Bartels, L. Ma, H. Haberland, and B. v.Issendorff, Phys. Rev. Lett. 103, 073401 (2009).

${ }^{16}$ J. Vigué, P. Labastie, and F. Calvo, Eur. Phys. J. D 8, 265 (2000).

${ }^{17}$ A. B. Nadykto and F. Yu, J. Geophys. Res. 108, 4717 (2003).

${ }^{18}$ T. Orii, Y. Okada, K. Takeuchi, M. Ichihashi, and T. Kondow, J. Chem. Phys. 113, 8026 (2000).

${ }^{19}$ T. Su, E. C. F. Su, and M. T. Bowers, J. Chem. Phys. 69, 2243 (1978).

${ }^{20}$ E. V. Tsiper, Phys. Rev. Lett. 94, 013204 (2005).

${ }^{21}$ S. L. Shostak, W. L. Ebenstein, and J. S. Muenter, J. Chem. Phys. 94, 5875 (1991).

${ }^{22}$ G. Kummerlöwe and M. K. Beyer, Int. J. Mass Spectrom. 244, 84 (2005).

${ }^{23}$ R. Levine and R. Bernstein, Molecular Reaction Dynamics and Chemical Reactivity (Oxford University Press, 1987).

${ }^{24}$ J. Brudermann, P. Lohbrandt, U. Buck, and V. Buch, J. Chem. Phys. 112, 11038 (2000).

${ }^{25}$ A. Glebov, A. P. Graham, A. Menzel, J. P. Toennies, and P. Senet, J. Chem. Phys. 112, $11011(2000)$.

${ }^{26}$ A. K. Soper and C. J. Benmore, Phys. Rev. Lett. 101, 065502 (2008). 